\title{
The limits of complex partial nephrectomy: are there any?
}

\author{
Vignesh T. Packiam, R. Houston Thompson
}

Department of Urology, Mayo Clinic, Rochester, MN, USA

Correspondence to: R. Houston Thompson. Department of Urology, Mayo Clinic, 200 1st St. SW, Gonda Building 7 South, Rochester, MN 55905, USA. Email: thompson.robert@mayo.edu.

Provenance: This is an invited article commissioned by the Section Editor Dr. Xiao Li (Department of Urology, Jiangsu Cancer Hospital, Jiangsu Institute of Cancer Research, Nanjing Medical University Affiliated Cancer Hospital, Nanjing, China).

Comment on: Beksac AT, Okhawere KE, Elbakry AA, et al. Management of high complexity renal masses in partial nephrectomy: A multicenter analysis. Urol Oncol 2019;37:437-44.

Submitted Sep 05, 2019. Accepted for publication Sep 18, 2019.

doi: $10.21037 / \mathrm{atm} .2019 .09 .97$

View this article at: http://dx.doi.org/10.21037/atm.2019.09.97

We read with great interest the recent publication by Beksac et al. that explored outcomes following robotic partial nephrectomy (RPN) for anatomically complex, defined as R.E.N.A.L. nephrometry score $\geq 10$, cT1-cT2 renal masses (1). The authors aimed to determine the relationship between baseline clinicopathologic characteristics with "trifecta" achievement, defined as warm ischemia time $<25$ minutes, lack of perioperative complications, and negative surgical margins (1). Using a multi-institutional dataset, they found that the trifecta was achieved in $62 \%$ of patients and that no preoperative baseline, clinical, or tumor characteristics were associated with trifecta achievement. Interestingly, only pathologic tumor stage was significantly associated with achievement of trifecta. This manuscript contributes to the still developing literature for the important topic regarding the role of partial nephrectomy for large and complex renal masses.

First, it should be noted that the overall perioperative outcomes from this cohort are quite impressive, particularly from a purely robotic cohort of anatomically complex partial nephrectomy patients. Beksac et al. report overall post-operative and high-grade complication rates of $10.4 \%$ and $2.3 \%$, respectively. This highlights the high volume and experience of the authors. A "benchmark" for outcomes of partial nephrectomy can be challenging to identify. In fact, a recent meta-analysis by Mir et al. that compared partial nephrectomy versus radical nephrectomy for $\mathrm{cT} 1 \mathrm{~b}$ and $\mathrm{cT} 2$ renal tumors was unable to control for tumor complexity when reporting complications (2). Kopp et al. quantified both renal-functional and oncologic outcomes for patients with cT1b or cT2 tumors in a set of publications, finding overall and high-grade complication rates of $37.5 \%$ and $17.5 \%$, which were considerably higher than in this publication by Beksac et al. $(3,4)$. Another recent report of complex renal masses with R.E.N.A.L. nephrometry score $>9$ by Garisto and colleagues demonstrated overall complication rates of $42 \%$ and $28 \%$ and major complication rates of $12 \%$ and $7 \%$ for open and RPN, respectively (5). As the authors themselves state as a limitation, their excellent outcomes for complex renal masses may not be applicable and generalizable to other centers. Furthermore, these results may not be applicable for open partial nephrectomy, which is commonly performed for complex renal masses.

Next, the methodology of this publication has a relatively unique focus compared to prior reports for large or complex renal masses. Rather than initially selecting patients by size or clinical tumor stage cutoffs, this report includes a range of tumor sizes and focuses on tumor complexity as measured by the R.E.N.A.L. nephrometry score. Ultimately, the implied goal of this manuscript is to establish safety and feasibility of partial nephrectomy in this setting relative to radical nephrectomy. The meta-analysis by Mir et al. focused on clinical T1b and T2 renal masses, concluding that partial nephrectomy confers comparable morbidity, renal functional, and oncologic outcomes relative to radical nephrectomy (2). The EORTC trial published by Van Poppel et al. showed no difference in overall survival between partial nephrectomy and radical nephrectomy for renal cell carcinoma patients, although tumor complexity was not controlled for (6). Importantly, a recent propensity- 
score adjusted study by Gershman et al. showed that partial nephrectomy ultimately did not result in overall survival benefit compared to radical nephrectomy in patients with cT1 renal cell carcinoma (7). An interesting additional analysis that could aid in this controversy and may be performed utilizing Beksac et al.'s database could assess outcomes of all cT1 and cT2 tumors and stratify outcomes by R.E.N.A.L. nephrometry score $\geq 10$. Such an analysis could ultimately allow for further association of R.E.N.A.L. nephrometry score complexity with outcomes, allowing for statistical control of confounding variables.

The choice of achievement of a "trifecta" in this publication warrants mention of several interesting points. First, the definition of trifecta for partial nephrectomy has not been standardized, with varying definitions noted in previously published literature $(8,9)$. While the definition in this publication is relatively commonly utilized, trifecta is generally described to assess the learning curve for minimally invasive partial nephrectomy (10). Several considerations that could improve using trifecta as an outcome would be: (I) to report each constituent of the trifecta separately as an outcome, (II) consider replacement of warm ischemia time $<25$ minutes with early and late post-operative renal-functional outcomes, and (III) consider replacement of surgical margins with recurrence free survival and cancer-specific survival. Although warm ischemia time $<25$ minutes and surgical margins have been established as reasonable surrogates for post-operative renal function and oncologic outcomes, in our opinion they are not as ideal as utilizing these outcomes themselves, especially if available within the dataset $(11,12)$.

In summary, determination of the appropriateness of partial nephrectomy for complex renal masses requires data regarding the perioperative, renal functional, and oncologic outcomes of this approach, which this publication contributes data for. The authors demonstrated that in experienced hands, the trifecta of warm ischemia time $<25$ minutes, lack of perioperative complications, and negative surgical margins could be achieved in $62 \%$ of patients, which was noteworthy in their highly anatomically complex renal mass series. However, further studies are needed to accurately predict the achievement of longer-term renal-functional and oncologic outcomes in patients undergoing partial nephrectomy for complex renal masses.

\section{Acknowledgments}

None.

\section{Footnote}

Conflicts of Interest: The authors have no conflicts of interest to declare.

Ethical Statement: The authors are accountable for all aspects of the work in ensuring that questions related to the accuracy or integrity of any part of the work are appropriately investigated and resolved.

\section{References}

1. Beksac AT, Okhawere KE, Elbakry AA, et al. Management of high complexity renal masses in partial nephrectomy: A multicenter analysis. Urol Oncol 2019;37:437-44.

2. Mir MC, Derweesh I, Porpiglia F, et al. Partial Nephrectomy Versus Radical Nephrectomy for Clinical T1b and T2 Renal Tumors: A Systematic Review and Meta-analysis of Comparative Studies. Eur Urol 2017;71:606-17.

3. Kopp RP, Mehrazin R, Palazzi KL, et al. Survival outcomes after radical and partial nephrectomy for clinical T2 renal tumours categorised by R.E.N.A.L. nephrometry score. BJU Int 2014;114:708-18.

4. Kopp RP, Liss MA, Mehrazin R, et al. Analysis of Renal Functional Outcomes After Radical or Partial Nephrectomy for Renal Masses $\geq 7 \mathrm{~cm}$ Using the RENAL Score. Urology 2015;86:312-9.

5. Garisto J, Bertolo R, Dagenais J, et al. Robotic versus open partial nephrectomy for highly complex renal masses: Comparison of perioperative, functional, and oncological outcomes. Urol Oncol 2018;36:471.e1-9.

6. Van Poppel H, Da Pozzo L, Albrecht W, et al. A prospective, randomised EORTC intergroup phase 3 study comparing the oncologic outcome of elective nephronsparing surgery and radical nephrectomy for low-stage renal cell carcinoma. Eur Urol 2011;59:543-52.

7. Gershman B, Thompson RH, Boorjian SA, et al. Radical Versus Partial Nephrectomy for cT1 Renal Cell Carcinoma. Eur Urol 2018;74:825-32.

8. Yerram NK, Dagenais J, Bryk DJ, et al. Trifecta Outcomes in Multifocal Tumors: A Comparison Between Robotic and Open Partial Nephrectomy. J Endourol 2018;32:615-20.

9. Tufek I, Mourmouris P, Doganca T, et al. Robot-Assisted Partial Nephrectomy for T1b Tumors: Strict Trifecta Outcomes. JSLS 2017. doi: 10.4293/JSLS.2016.00113. 10. Larcher A, Muttin F, Peyronnet B, et al. The Learning 
Curve for Robot-assisted Partial Nephrectomy: Impact of Surgical Experience on Perioperative Outcomes. Eur Urol 2019;75:253-6.

11. Thompson RH, Lane BR, Lohse CM, et al. Renal Function After Partial Nephrectomy: Effect of Warm Ischemia Relative to Quantity and Quality of Preserved

Cite this article as: Packiam VT, Thompson RH. The limits of complex partial nephrectomy: are there any? Ann Transl Med 2019;7(Suppl 8):S345. doi: 10.21037/atm.2019.09.97
Kidney. Urology 2012;79:356-60.

12. Shah PH, Moreira DM, Okhunov Z, et al. Positive Surgical Margins Increase Risk of Recurrence after Partial Nephrectomy for High Risk Renal Tumors. J Urol 2016;196:327-34. 